\title{
Effect of Slice Thickness Reconstruction on Quantitative Vascular Parameters in Perfusion CT
}

\author{
Anxo Martínez-de-Alegría ${ }^{*}$, Roberto García-Figueiras ${ }^{1}$, Sandra Baleato-González ${ }^{1}$, \\ María José Rodriguez-Alvarez², Iago Martínez-Souto3 ${ }^{3}$, Carmen Villalba-Martín' \\ ${ }^{1}$ Departments of Radiology, Hospital Clínico Universitario, Santiago de Compostela, Spain \\ ${ }^{2}$ Statistics, Hospital Clínico Universitario, Santiago de Compostela, Spain \\ ${ }^{3}$ General Electric, Santiago de Compostela, Spain \\ Email: *anxomartinezdealegria@gmail.com
}

Received 18 November 2013; revised 18 December 2013; accepted 26 December 2013

Copyright (C) 2014 by authors and Scientific Research Publishing Inc.

This work is licensed under the Creative Commons Attribution International License (CC BY).

http://creativecommons.org/licenses/by/4.0/

(c) (i) Open Access

\section{Abstract}

Objective: The purpose of this study was to determine how the slice thickness reconstruction influences quantitative perfusion CT parameters. Materials and Methods: Eighteen patients with cancer (15 non-small-cell lung cancer, 2 rectal cancer and 1 renal cancer) were examined prospectively with multidetector row CT. A 90-second perfusion study was performed after intravenous bolus injection of contrast material. Blood flow, blood volume, mean transit time and permeability-surface area product were determined at three different slice thickness reconstruction (1.25, 2.5 and $5 \mathrm{mms}$ ) both in tumors and in paraspinal muscle. Mean values, limits of agreement between measurements and within-subject coefficient of correlation were obtained for these thicknesses. Results: Mean \pm standard deviation BF, BV, MTT and PS in lesions were $118.7 \pm 117.9$ $\mathrm{mL} / \mathrm{min} / 100 \mathrm{~g}$ tissue, $8.2 \pm 8.2 \mathrm{~mL} / 100 \mathrm{~g}$ tissue, $7.5 \pm 5.4$ seconds and $10.3 \pm 7.2 \mathrm{~mL} / \mathrm{min} / 100 \mathrm{~g}$ tissue respectively at $1.25 \mathrm{~mm}$ slice thickness; $116.1 \pm 115.7 \mathrm{~mL} / \mathrm{min} / 100 \mathrm{~g}$ tissue, $7.8 \pm 8.7 \mathrm{~mL} / 100 \mathrm{~g}$ tissue, $7 \pm 4.5$ seconds and $10.4 \pm 7.5 \mathrm{~mL} / \mathrm{min} / 100 \mathrm{~g}$ tissue at $2.5 \mathrm{mms}$; and $119.6 \pm 115.7$ $\mathrm{mL} / \mathrm{min} / 100 \mathrm{~g}$ tissue, $7.8 \pm 8.8 \mathrm{~mL} / 100 \mathrm{~g}$ tissue, $5.4 \pm 3.4$ seconds and $9.6 \pm 7.5 \mathrm{~mL} / \mathrm{min} / 100 \mathrm{~g}$ tissue at $5 \mathrm{mms}$. Differences between means for different slice thickness where relatively small in all parameters $(<15 \%)$ except in MTT where difference was up to $37 \%$. 95\% limits of agreement were worse when comparing more different slice thicknesses (e.g. $1.25 \mathrm{vs} 5 \mathrm{mms}$ ) than when comparing more close slice thicknesses (1.25 vs $2.5 \mathrm{mms}$ or $2.5 \mathrm{vs} 5 \mathrm{mms}$ ). Conclusions: There is a significant variability in perfusion parameter measurements at different slice thickness reconstruction, particularly in MTT. The more close together the slice thicknesses were, the smaller was the variability.

*Corresponding author. 


\section{Keywords}

\section{Tomography; Neoplasms; Blood Supply; Diagnostic Imaging}

\section{Introduction}

Computed tomography has become the main diagnostic tool in staging and monitoring the response to therapy of many tumours due to its relative low cost and wide availability. Perfusion CT is a rapidly developing technique that allows functional evaluation of tissue vascularity [1]. It was developed 30 years ago, primarily for quantification of cerebral blood perfusion in patients with acute stroke [2], but the recent availability of MDCT scanners with a higher number of detector rows that allow greater anatomic coverage and higher temporal resolution, has rediscovered the technique as a promising noninvasive tool for evaluation of the microcirculatory changes associated with several neoplasms. The increasing interest in oncologic applications of perfusion CT has been fueled by the widely recognized limitations of morphologic assessment of treatment response. These limitations are particularly evident with novel antiangiogenic agents, that are tipically not citotoxic but instead produce disease stabilization [3].

Perfusion CT has been validated against histologic markers of angiogenesis in a variety of cancers [4] and can be considered a surrogated imaging biomarker for tumor angiogenesis. Reported clinical uses of perfusion CT include differentiation between benign and malignant lesions, tumor characterization, prognostic biomarker and monitoring therapy.

In most publishes studies on perfusion CT, authors almost universally recommend slice thickness reconstructions of not less than $5 \mathrm{~mm}$ in their protocols, which reportedly guarantees a correct balance between the requirements for spatial resolution and the signal-to-noise ratio [5]. But there are several clinical settings were slice thickness reconstructions of less than 5 mms may be needed, such as when non-axial multiplanar reconstructions are to be performed or when small anatomic structures like adenopathies need to be studied. The primary aim of this study was to evaluate the effect of slice thickness reconstruction on quantitative vascular parameters in perfusion CT scan.

\section{Materials and Methods}

\subsection{Patients and Target Lesions}

Our study group include 18 oncological patients with solid lesions that underwent perfusion CT as part of a research project on multiparametric CT evaluation of thoracic and abdominal tumors, conducted by two of us (A. M-de-A, R.G-F.) at the Hospital Clínico Universitario of Santiago de Compostela, Spain. One patient was excluded because of movement during the CT adquisition. Target lesions were required to be contrast enhanced solid masses larger than $2 \mathrm{cms}$ in the longest diameter.

This study was performed with the approval of the local Medical Ethics Committee. The patients were informed of the investigational nature of the study and written informed consent was obtained from all of them.

\subsection{Computed Tomographic Perfusion Technique}

Perfusion examinations were performed using a 64-row spiral CT unit (LightSpeed VCT; General Electric Medical Systems, Milwakee, USA).

A preliminary low dose 5-mm-thick CT scan without contrast agent was performed to obtain a panoramic view of the anatomical region of study (thorax in lung cancer patients, abdomen in cases of renal cancer and pelvis in rectal cancer). A variable scanning volume was selected for the dynamic CT, depending on the size of the tumor to be studied. The anatomic coverage ranged from $40 \mathrm{mms}$ (single-section data acquisition) in 7 patients, to up to $110 \mathrm{mms}$ (table-togging) in 10 patients.

Dynamic study was performed with low dose $(80 \mathrm{kv}, 120 \mathrm{mAs})$ and rotation time $0.4 \mathrm{sec}$. The contrast agent was injected via a 18-to-20 cannula in the antecubital vein with a power injector (Medrad, Pittsburg, USA). Scanning was initiated $5 \mathrm{~s}$ after the intravenous rapid infusion (injection rate of $5 \mathrm{ml} / \mathrm{s}$ ) of $50 \mathrm{ml}$ of non-ionic io- 
dinated contrast material (Optiray $300 \mathrm{mg} / \mathrm{dl}$. Mallinckrodt Medical Imaging), followed by $40 \mathrm{ml}$ of saline at 3 $\mathrm{ml} / \mathrm{sec}$. The dynamic CT data were acquired every 1 second for 35 seconds, during breath holding at the end of inhalation; and then, an additional five series of data were obtaining with breath holding at the end of inhalation-at 40, 50, 60 and 90 seconds. By applying an iterative image reconstruction algorithm, relative low radiation doses can be administered mantaining a good signal-to-noise ratio.

After finishing the acquisition of data, images were reconstructed at $1.2 \mathrm{~mm}, 2.5 \mathrm{~mm}$ and $5 \mathrm{~mm}$ slice thickness with a soft reconstruction kernel (Standard Recon type), thus generating 3 separate sets of dynamic contrast enhanced images which could then be loaded into the workstation.

\subsection{Image Data Analysis}

The images and data obtained were anonymized and transferred to an image-processing workstation (Advanced Windows 4.6; General Electric Company) where was analyzed by a thoracic radiologist with 2 years of experience in perfusion CT. Commercially available software (CT perfusion 4D; General Electric Company) was used for perfusion CT analysis. A region of interest (ROI) was placed in the aorta for calculation of the arterial input. The software use a deconvolution model for automated quantification of tissue perfusion, generating parametric maps of blood flow (BF), blood volume (BV), mean transit time (MTT) of the contrast medium and permeability surface-area product (PS), wich measures contrast medium extravasation into the extravascular space. In every parametric map, two circular ROIs were placed (area range, 30 to $78 \mathrm{~mm}^{2}$ )—one on the center of the lesion studied and other on the paravertebral musculature region-obtaining for each ROI area the values of BF (in millimeters per $100 \mathrm{mg}$ per minute), BV (in millimeters per $100 \mathrm{mg}$ ), MTT (in seconds) and PS (in millimeters per $100 \mathrm{mg}$ per minute). The two ROIs drawn in every parametric map were copied with identical size and position to the three different slice thickness: 1.2, 2.5 and $5 \mathrm{~mm}$, obtaining a set of 6 different values for each parameter and patient ( 3 for the lesion and 3 for the muscle, that is used as an internal reference control).

\subsection{Statistical Analysis}

A descriptive analysis of BF, BV, MTT, and PS parameters was firstly performed. For each slice thickness (1.25, 2.5 and $5 \mathrm{~mm}$ ), and for lesion and muscle separately, results are presented as the mean and standard deviation.

A Bland and Altman analysis [6] was then performed to obtain the limits of agreement (LoA) between measurements obtained at different slice thickness. For this purpose, the analyses were done separately in lesion and muscle, and the following comparisons were carried out for each parameter: $1.25 \mathrm{~mm}$ versus $2.5 \mathrm{~mm}, 1.25 \mathrm{~mm}$ versus $5.0 \mathrm{~mm}$; and, $2.5 \mathrm{~mm}$ versus $5.0 \mathrm{~mm}$. In none of the analyses performed, the results showed that differences between paired measurements significantly increased with increasing values of the parameter. Acordingly, no transformation was applied to the data.

Finally, we also assesed the reproducibility of measurements obtained at different slice thickness with the within-subject coefficient of correlation, jointly with the corresponding 95\% confidence interval [7]. To this aim, a random effect model was fitted for each parameter, for muscle and lesion separately, and for each pair of slice thickness. Again, no data transformation was applied.

All analyses were conducted by using R software [8]. Bland-Altman analysis was performed by using the MethComp package [9], and random effect models were fitted using the nlme package [10].

\section{Results}

In total, 18 patients had evaluable imaging data. The mean age was 63 years (range 23 - 92 years). The primary tumors were 15 non small cell lung cancer, 2 rectal carcinoma and 1 renal carcinoma. There were 13 men (mean age 59 years) and 5 women (mean age 75 years).

Table 1 summarizes the mean and standard deviation of the three sets of measurements corresponding to different slice thickness in tumor and muscle. The mean values obtained in the paraspinous muscles were significantly lower when compared with the tumor tissue for all perfusion parameters, except for the MTT where the values were higher in muscles than in tumor.

The mean CT perfusion values were affected by the thickness of the slices. Differences between means for different slice thickness were relatively small in all parameters (under 15\%) except in MTT, were means varied from 7.5 secs at 1.25 mms to 5.45 secs at 5 mms in tumours (a difference of up to 37\%), and from 11.2 secs at 
Table 1. Characteristics of the sample.

\begin{tabular}{cccc}
\hline \multirow{2}{*}{ Parameter } & \multicolumn{3}{c}{ Slice thickness (mm) } \\
\cline { 2 - 4 } & $\mathbf{1 . 2 5}$ & $\mathbf{2 . 5}$ & $\mathbf{5}$ \\
BF (mL/min/100g) & \multicolumn{2}{c}{ Lesion } \\
BV (mL/100g) & $118.772(117.986)^{\mathrm{a}}$ & $116.140(115.744)$ & $119.629(115.794)$ \\
MTT (sec) & $8.257(8.245)$ & $7.827(8.706)$ & $7.806(8.849)$ \\
PS (mL/min/100g) & $7.510(5.492)$ & $7.070(4.501)$ & $5.457(3.427)$ \\
& $10.370(7.294)$ & $10.435(7.567)$ & $9.609(7.553)$ \\
BF (mL/min/100g) & Muscle & & $20.978(28.065)$ \\
BV (mL/100g) & $20.345(26.624)$ & $20.382(23.224)$ & $1.642(1.834)$ \\
MTT (sec) & $1.776(1.445)$ & $1.885(1.492)$ & $8.404(4.798)$ \\
PS (mL/min/100g) & $10.347(4.949)$ & $11.282(4.807)$ & $3.889(4.614)$ \\
\hline
\end{tabular}

${ }^{\mathrm{a}}$ Mean (Standard Deviation).

$2.5 \mathrm{mms}$ and 8.4 secs at $5 \mathrm{mms}$ in muscle (a difference of up to 34\%). Parameters for different perfusion parameters of lesion and muscle are presented in Figure 1.

Table 2 summarizes the mean differences and 95\% limits of agreement between the sets of measurements obtained at different slice thickness. The data suggest agreement in tumor tissue was worse when comparing 1.25 mms versus 5 mms than when comparing 1.25 vs $2.5 \mathrm{mms}$ or 2.5 vs $5 \mathrm{mms}$ : in other words, the more different the slice thickness are, the worse is the agreement between the values registered in the same ROI. Regarding to muscle values, limits of agreement between different thickness were in general smaller than in lesions, and seemed not to be related with the degree of separation between the slice thicknesses. Overall, tumor blood volume and permeability surface are the least variable parameters, both in lesions and in muscle.

Table 3 shows that the coefficient of variation within-subject for lesions was higher when comparing perfusion values at separate thicknesess like 1.25 vs 5 mms (28.1\% for BF, $28.9 \%$ for BV, $60.6 \%$ for MTT and 31.5\% for PS), than when comparing more close thicknesess like 1.25 vs $2.5 \mathrm{mms}$ or 2.5 vs $5 \mathrm{mms}$. Overall, the coefficients of variation between different slice thicknesses obtained for MTT were higher than those obtained for the rest of parameters, both in tumors (42\% for 2.5 vs $5 \mathrm{mms}$ and $60.6 \%$ for $1.25 \mathrm{vs} 2.5 \mathrm{mms}$ ) and in muscle (46.2\% for 2.5 vs $5 \mathrm{mms}$ and $47.1 \%$ for $1.25 \mathrm{vs} 5 \mathrm{mms}$ ).

\section{Discussion}

Although helical perfusion CT have been used as a clinical technique for several years, the effect of slice thickness reconstruction on quantitative vascular parameters has not been fully studied. Most of the reported protocols suggest that slice thickness reconstruction of less than $5 \mathrm{mms}$ is not advisable, because the resulting increased spatial resolution is obtained at the expenses of a decreasing in the signal-to-noise ratio. But to our knowledge, there are no studies assessing the variability of perfusion CT measurements obtained at different slice thickness reconstruction and their impact on the clinical use of the technique.

A variety of factors contribute to measurement variability, being generally divided into intrinsic and extrinsic factors. Intrinsic factors include tumor heterogeneity (that may represent up to $25 \%$ of noise measurement [11]), physiologic components like cardiac output, and intrinsic variability of the technique. Extrinsic factors include observer variability and variability related to adquisition parameters, specially slice collimation [12]. Although it is well known that the image noise is dependent mainly on the slice thickness-from the same CT source data, thicker slices are less noisy than thinner reconstructions-our study focuses not on the signal-to-noise ratio of the image but in the variability of permeability measurements due to the slice thickness reconstruction.

Reproducibility studies involving normal brain, colorectal carcinoma, lung carcinoma and carcinoma hepatocellular have shown good correlation for perfusion CT, with a variability of 13\% to 30\% [1]. In a CT perfusion reproducibility study using two measurement sets obtained 48 hours apart in patients with colorectal cancer, Goh et al. [12] found mean difference an $95 \%$ limits of agreement of $8.80(-50.5,68) \mathrm{mL} / \mathrm{g}$ tissue for BF, -0.04 $(-2.5,2.42) \mathrm{mL} / 100 \mathrm{~g}$ tissue for BV, $-0.99(-8.19,6.20)$ secs for MTT and $1.20(-5.42,7.82) \mathrm{mL} / 100 \mathrm{~g}$ tissue/min for PS. These $95 \%$ limits of agreement reflect the intrinsic variability of the technique and were con- 

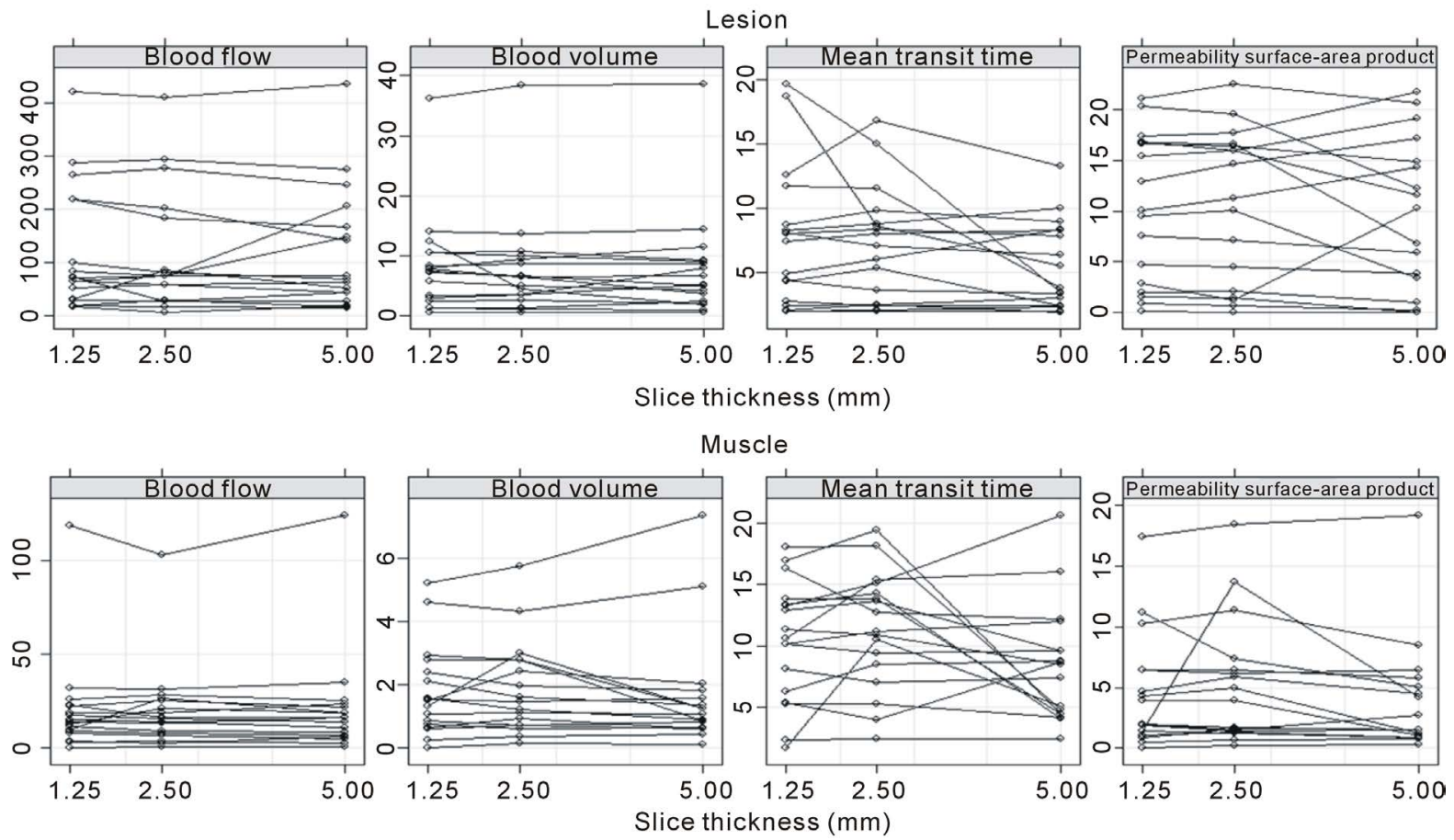

Figure 1. Graphs show changes in CT perfusion parameters between measurements at different slice thickness for lesion and paravertebral musculature region.

Table 2. Bland-Altman analysis. Limits of agreement.

\begin{tabular}{|c|c|c|c|}
\hline Parameter & 1.25 vs 2.5 & 2.5 vs 5.0 & 1.25 vs 5.0 \\
\hline \multicolumn{4}{|c|}{ Lesion } \\
\hline $\mathrm{BF}(\mathrm{mL} / \mathrm{min} / 100 \mathrm{~g})$ & $2.63(-40.37,45.63)^{*}$ & $-3.49(-90.47,83.49)$ & $-0.86(-98.67,96.96)$ \\
\hline BV (mL/100g) & $0.43(-3.79,4.65)$ & $0.02(-3.50,3.54)$ & $0.45(-6.26,7.16)$ \\
\hline MTT (sec) & $0.44(-5.56,6.44)$ & $1.61(-5.30,8.53)$ & $2.05(-8.60,12.71)$ \\
\hline PS (mL/min/100g) & $-0.06(-1.73,1.60)$ & $-0.83(-8.33,9.98)$ & $0.76(-8.30,9.82)$ \\
\hline \multicolumn{4}{|c|}{ Muscle } \\
\hline $\mathrm{BF}(\mathrm{mL} / \mathrm{min} / 100 \mathrm{~g})$ & $-0.04(-12.82,12.5)$ & $-0.60(-12.84,11.64)$ & $-0.63(-9.08,7.81)$ \\
\hline BV (mL/100g) & $-0.11(0.97,-1.19)$ & $0.24(-1.47,1.96)$ & $0.13(-1.65,1.92)$ \\
\hline MTT (sec) & $-0.94(-6.44,4.57)$ & $2.88(-9.00,14.5)$ & $1.94(-10.30,14.18)$ \\
\hline PS (mL/min/100g) & $-0.73(-7.13,5.66)$ & $1.29(-3.77,6.36)$ & $0.58(-3.52,4.64)$ \\
\hline
\end{tabular}

${ }^{*}$ Average difference (Limits of Agreement-LoA).

Table 3. Within-subject coefficient of variation.

\begin{tabular}{|c|c|c|c|}
\hline \multirow{2}{*}{ Parameter } & \multicolumn{3}{|c|}{ Within-subject CV (\%) ${ }^{*}$} \\
\hline & 1.25 vs 2.5 & 2.5 vs 5.0 & 1.25 vs 5.0 \\
\hline \multicolumn{4}{|c|}{ Lesion } \\
\hline $\mathrm{BF}$ (mL/min/100g) & $12.65(9.35,15.97)$ & $25.39(18.87,31.92)$ & $28.15(20.94,35.36)$ \\
\hline BV (mL/100g) & $18.41(7.59,29.23)$ & $15.43(5.94,24.93)$ & $28.92(11.94,45.91)$ \\
\hline MTT (sec) & $28.57(15.64,41.5)$ & $42.01(24.01,60.01)$ & $60.63(34.69,86.58)$ \\
\hline PS (mL/min/100g) & $5.51(2.92,8.1)$ & $31.86(16.82,46.9)$ & $31.56(16.78,46.36)$ \\
\hline \multicolumn{4}{|c|}{ Muscle } \\
\hline $\mathrm{BF}(\mathrm{mL} / \mathrm{min} / 100 \mathrm{~g})$ & $21.54(7.45,35.63)$ & $20.40(6.9,33.91)$ & $14.19(4.32,24.05)$ \\
\hline BV (mL/100g) & $20.61(11.27,25.73)$ & $34.71(28.69,63.83)$ & $36.22(29.01,65.24)$ \\
\hline MTT (sec) & $18.50(15.17,21.83)$ & $46.26(38.16,54.36)$ & $47.12(38.77,55.47)$ \\
\hline PS (mL/min/100g) & $46.79(20.68,72.9)$ & $43.28(17.93,68.63)$ & $34.87(13.81,55.93)$ \\
\hline
\end{tabular}

* Data in parentheses are 95\% confidence intervals. 
sidered by the authors acceptable for all perfusion parameters and suitable for day to day therapeutic assessment. In our study, the 95\% limits of agreement obtained represent the boundaries within which the difference between means of two different thicknesses is expected to lie $95 \%$ of the time: the narrower the limits, the lesser the variability. Our results are not very different from Goh's data, showing that the variability related to extrinsic factors like slice thickness reconstruction may be similar to intrinsic measurement variability of the technique. Our data suggest that tumor BV and PS are the least variable parameters.

The absolute values of CT perfusion parameters are also affected by the method of analysis. By addition of the second phase (intermittent cine) to the initial cine adquisition, $\mathrm{Ng}$ et al. [13] obtained differences between means of up to $11 \%, 23 \%, 21 \%$ and $138 \%$ for BF, BV, MTT and PS respectively. Comparing several slice thickness reconstructions we found differences less than $15 \%$ for all parameters except for MTT, where differences may be as high as $37.7 \%$. Also, measurements obtained with different software may not be directly interchangeable, as proved by Goh et al. [14]. Using two different commercially available CT perfusion software packages, they found a mean difference and $95 \%$ limits of agreement of $-3.6 \mathrm{~mL} / 100 \mathrm{~mL}(-18.4$ to 11.2 $\mathrm{mL} / 100 \mathrm{~mL})$ for PS and $-3.9 \mathrm{~mL} / 100 \mathrm{~mL}(-10.9$ to $3.0 \mathrm{~mL} / 100 \mathrm{~mL})$ for BV. Agreement between measurements was worse for BV (coefficient of variation 46.5\%) than for PS (coefficient of variation 37.4\%), results described by the authors as only "moderated agreement". In our data, the highest coefficients of variation were found in MTT, both in lesions (28.5\%, 42\% and 60.6\%) and in muscle (18.5\%, 46.2\% and 47.1\%).

There were some limitations of our study. First, although we have established that the variability of perfusion parameters at different thickness reconstruction may be similar than the one induced by other factors that contribute to measurement variability, the study does not suggest any explanation for this variability being higher in some parameters (PS and MTT) than in others (BV and BF). On the other hand, the series is limited and further studies on this topic are needed.

\section{Conclusion}

We found that absolute values of CT perfusion parameters were markedly influenced by slice thickness reconstruction, with a higher variability found in MTT. But this lack of agreement fell into the range of variability induced by other intrinsic and extrinsic factors described in literature. The use of the same slice thickness reconstruction for all patients is advisable in order to avoid a significant source of measurement error in clinical routine.

\section{References}

[1] Kambadakone, A.R. and Sahani, D.V. (2009) Body Perfusion CT: Technique, Clinical Applications, and Advances. Radiologic Clinics of North America, 47, 161-178. http://dx.doi.org/10.1016/j.rcl.2008.11.003

[2] Axel, L. (1980) Cerebral Blood Flow Determination by Rapid-Sequence Computed Tomography: Theoretical Analysis. Radiology, 137, 679-686.

[3] García-Figueiras, R., Goh, V.J., Padhani, A.R., Baleato-Gonzalez, S., Garrido, M., León, L. and Gómez-Caamaño, A. (2013) CT Perfusion in Oncologic Imaging: A Useful Tool? AJR, 1, 8-19. http://dx.doi.org/10.2214/AJR.11.8476

[4] Ng, Q.S. and Goh, V. (2010) Angiogenesis in Non-Small Cell Lung Cancer: Imaging with Perfusion Computed Tomography. Journal of Thoracic Imaging, 25, 142-150. http://dx.doi.org/10.1097/RTI.0b013e3181d29ccf

[5] Petralia, G., Bonello, L., Viotti, S., Preda, L., d’Andrea G. and Bellomi, M. (2010) CT Perfusion in Oncology: How to Do It. Cancer Imaging, 10, 8-19. http://dx.doi.org/10.1102/1470-7330.2010.0001

[6] Bland, J.M. and Altman, D.G. (1986) Statistical Methods for Assessing Agreement between Two Methods of Clinical Measurement. Lancet, 1, 307-310. http://dx.doi.org/10.1016/S0140-6736(86)90837-8

[7] Quan, H. and Shih, W.J. (1996) Assessing Reproducibility by the Within-Subject Coefficient of Variation with Random Effects Models. Biometrics, 52, 1195-1203. http://dx.doi.org/10.2307/2532835

[8] R Core Team (2013) R: A Language and Environment for Statistical Computing. http://www.R-project.org/

[9] Carstensen, B., Gurrin, L. and Ekstrom, C. (2012) MethComp: Functions for Analysis of Method Comparison Studies. http://bendixcarstensen.com/MethComp/

[10] Pinheiro, J., Bates, D., DebRoy, S., Sarkar, D. and the R Development Core Team (2013) nlme: Linear and Nonlinear Mixed Effects Models. R package version 3.1-109.

[11] Goh, V., Dattani, M., Farwell, J., Shekhdar, J., Tam, E., Patel, S., Juttla, J., Simcock, I., Stirling, J., Mandeville, H., 
Aird, E. and Hoskin, P. (2011) Radiation Dose from Volumetric Helical Perfusion CT of the Thorax, Abdomen or Pelvis. European Radiology, 21, 974-981. http://dx.doi.org/10.1007/s00330-010-1997-y

[12] Goh, V., Halligan, S., Hugill, J.-A. and Bartram, C.I. (2006) Quantitative Assessment of Tissue Perfusion Using MDCT Comparison of Colorectal Cancer and Skeletal Muscle Measurement Reproducibility. AJR, 187, pp.164-169. http://dx.doi.org/10.2214/AJR.05.0050

[13] Ng, C.S., Chandler, A.G., Wei, W., Herron, D.H., Anderson, E.F., Kurzrock, R. and Charnsangavej, C. (2011) Reproducibility of CT Perfusion Parameters in Liver Tumors and Normal Liver. Radiology, 260, 762-770. http://dx.doi.org/10.1148/radiol.11110331

[14] Goh, V., Halligan, S. and Bartram, C.I. (2007) Quantitative Tumor Perfusion Assessment with Multidetector CT: Are Measurements from Two Commercial Software Packages Interchangeable? Radiology, 242, 777-782. http://dx.doi.org/10.1148/radiol.2423060279

\section{Abbreviations}

BF: blood flow

BV: blood volume

$\mathrm{CV}$ : coefficient of variation

LoA: limits of agreement

MTT: mean transit time

PS: permeability-surface area product

ROI: region of interest 